\title{
Chlorhexidine hexametaphosphate nanoparticles as a novel antimicrobial coating for dental implants
}

\author{
Natalie J. Wood ${ }^{1,2,3} \cdot$ Howard F. Jenkinson ${ }^{4} \cdot$ Sean A. Davis ${ }^{2} \cdot$ Stephen Mann $^{2}$ • \\ Dominic J. O'Sullivan' ${ }^{1}$ Michele E. Barbour ${ }^{1}$
}

Received: 8 January 2015/Accepted: 20 June 2015/Published online: 30 June 2015

(c) The Author(s) 2015. This article is published with open access at Springerlink.com

\begin{abstract}
Dental implants are an increasingly popular solution to missing teeth. Implants are prone to colonisation by pathogenic oral bacteria which can lead to inflammation, destruction of bone and ultimately implant failure. The aim of this study was to investigate the use of chlorhexidine (CHX) hexametaphosphate (HMP) nanoparticles (NPs) with a total CHX concentration equivalent to $5 \mathrm{mM}$ as a coating for dental implants. The CHX HMP NPs had mean diameter $49 \mathrm{~nm}$ and composition was confirmed showing presence of both chlorine and phosphorus. The NPs formed micrometer-sized aggregated surface deposits on commercially pure grade II titanium substrates following immersion-coating for $30 \mathrm{~s}$. When CHX HMP NP-coated titanium specimens were immersed in deionised water, sustained release of soluble CHX was observed, both in the absence and presence of a salivary pellicle, for the duration of the study (99 days) without reaching a plateau. Control specimens exposed to a solution of aqueous $25 \mu \mathrm{M}$ CHX (equivalent to the residual aqueous CHX present with the NPs) did not exhibit CHX release. CHX HMP NP-coated surfaces exhibited antimicrobial efficacy against oral primary colonising bacterium Streptococcus gordonii within
\end{abstract}

Michele E. Barbour

m.e.barbour@bristol.ac.uk

1 Oral Nanoscience, School of Oral \& Dental Sciences, University of Bristol, Bristol, UK

2 Centre for Organized Matter Chemistry, School of Chemistry, University of Bristol, Bristol, UK

3 Bristol Centre for Functional Nanomaterials, University of Bristol, Bristol, UK

4 Oral Microbiology, School of Oral \& Dental Sciences, University of Bristol, Bristol, UK
$8 \mathrm{~h}$. The antimicrobial efficacy was greater in the presence of an acquired pellicle which is postulated to be due to retention of soluble CHX by the pellicle.

\section{Introduction}

Titanium is the major component of most dental implant systems, since it exhibits excellent biocompatibility and supports osseointegration. The rate of osseointegration is affected by surface roughness; an implant with moderate roughness of $1-2 \mu \mathrm{m}$ is thought to be optimal [1]. With rougher implant surfaces comes an increased susceptibility to bacterial adhesion, at least in vitro [2-4], and this has been attributed to increased protection from shear forces [4]. Drawing inference from such in vitro data, it has been proposed that rough implant surfaces may exhibit a greater propensity for implant-associated infections in vivo [5]. Peri-implant mucositis, the most common form of infection, occurs in approximately $80 \%$ of subjects [6], resulting in reversible inflammation of peri-implant soft tissues. The more severe peri-implantitis occurs in about $28-56 \%$ of subjects [6] and, as well as soft tissue inflammation, results in loss of the supporting bone. Treatment of peri-implantitis has unpredictable outcomes and control of plaque in the mouth surrounding the implant is essential [7].

Upon exposure to the oral cavity, implants, like all materials, are rapidly coated with a thin proteinaceous film known as the salivary pellicle. This is composed primarily of salivary glycoproteins and mediates adhesion of oral primary colonisers [8]; the most common such species found on dental implants are the streptococci [9]. Once adhered, the bacteria proliferate and excrete an extracellular polysaccharide matrix which protects the developing microcolony. Secondary colonising bacteria then adhere to 
the primary colonisers resulting in biofilm formation. The microbiota found in infected implant sites have similarities to those seen in periodontitis [10], although with somewhat less diversity [11].

Chlorhexidine (CHX) is a broad spectrum antimicrobial and antifungal agent belonging to the biguanide class of drugs. It is used extensively in medicine and dentistry, as the soluble digluconate salt, for a wide range of applications including mouthrinses, eye drops and as a pre-surgical topical cleansing agent. Its non-specific mode of action is associated with rupture of the bacterial cell membrane resulting in leakage of intracellular components [12]. CHX adsorbs to titanium oxide surfaces, and the resultant CHXcoated surface can reduce growth of Streptococcus gordonii [13], but the CHX is rapidly depleted providing only a short-term antimicrobial effect [14].

Antimicrobial nanoparticles (NPs) offer a method for imparting antimicrobial properties to implant surfaces. One advantage of NPs as a coating for dental implants when not embedded in a film but applied directly to the titanium is that, with careful control of the doping and distribution, their small size provides the opportunity to leave the majority of the titanium surface uncoated and thus available for osteoblast colonisation and subsequent osseointegration. Elemental silver is used as an antimicrobial agent in many fields of medicine, and silver NPs embedded in various film coatings have been applied to titanium implant surfaces for the purpose of conferring antimicrobial activity [15-19]. However, cytotoxic effects of silver NPs have been reported and thus safety concerns persist [20, 21]. Chlorhexidine-hexametaphosphate (CHX-HMP) NPs have recently been reported and act as a slow release device for soluble CHX [22]. The research question was: do $\mathrm{CHX}-$ HMP NPs have potential as an antimicrobial coating for titanium dental implants. The hypothesis to be tested was: CHX-HMP NPs have no antimicrobial efficacy when used as a coating for titanium.

\section{Materials and methods}

\subsection{Nanoparticle synthesis, specimen preparation and characterisation}

$100 \mathrm{~mL} 10 \mathrm{mM}$ aqueous sodium HMP (Sigma-Aldrich Company Ltd, Dorset, UK) was added to $100 \mathrm{~mL} 10 \mathrm{mM}$ of aqueous CHX digluconate (Sigma-Aldrich Company Ltd, Dorset, UK) under constant stirring and ambient conditions. This resulted in a suspension of CHX-HMP $\mathrm{NPs}$, with a total concentration of $5 \mathrm{mM}$ of both $\mathrm{CHX}$ and HMP.

Approximately $20 \mu \mathrm{L}$ of NP suspension was deposited on a 200 mesh carbon-coated copper/gold grid (Agar
Scientific, Essex, UK), left undisturbed for $2 \mathrm{~min}$, then placed on filter paper to dry. Specimens were imaged using transmission electron microscopy (TEM; JEM 1200 EX MKI: Jeol, Welwyn Garden City, UK). In-situ energydispersive X-ray spectroscopy (EDX; ISIS 300: Oxford Instruments, Bristol, UK) was used to determine the elemental composition of the NP precipitate.

Square sections $(10 \times 10 \times 1 \mathrm{~mm})$ of grade 2 commercially pure titanium (Ti-TEK Ltd, Sutton Coldfield, UK) were polished using 120 grit silicon carbide paper, followed by $10 \mathrm{~min}$ ultrasonication in acetone and $10 \mathrm{~min}$ ultrasonication in industrial methylated spirits, before being allowed to dry in air. To coat the titanium specimens with nanoparticles, individual substrates were suspended in $200 \mathrm{~mL}$ of the colloidal suspension for $30 \mathrm{~s}$ during rapid stirring, followed by $10 \mathrm{~s}$ immersion in deionised water, before being blotted to remove excess water and allowed to dry in air. Selected specimens were coated with goldpalladium alloy, using a sputter coater (SC7620, Quorum Technologies, East Grinstead, UK) and imaged using scanning electron microscopy (SEM) (Phenom, PhenomWorld, Eindhoven, Netherlands).

\subsection{CHX elution from CHX-HMP NP coated titanium}

The release of soluble $\mathrm{CHX}$ from the CHX-HMP NP coated specimens was recorded as a function of time. The experiments were conducted with and without the application of a salivary pellicle to the NP-coated titanium, to determine whether $\mathrm{CHX}$ elution was impeded by the presence of a pellicle. Control groups were titanium substrates treated with deionised water or a $25 \mu \mathrm{M} \mathrm{CHX}$ solution (the residual $\mathrm{CHX}$ concentration in the NP suspension) instead of the NP suspension.

Titanium specimens for elution studies were prepared and coated with NPs as described above. Control specimens were immersed in deionised water for $40 \mathrm{~s}$ (deionised water controls) or immersed in $25 \mu \mathrm{M}$ CHX solution for $30 \mathrm{~s}$ exposure followed by $10 \mathrm{~s}$ in deionised water (aqueous CHX controls).

For those specimens to be coated with a salivary pellicle, stimulated saliva was collected from 5 donors (saliva bank REC reference: 08/H0606/87+5). The saliva was pooled and $0.02 \mathrm{w} / \mathrm{v} \%$ sodium azide was added to prevent bacterial growth. Titanium specimens were incubated, after coating (with NPs or control treatments), at $37{ }^{\circ} \mathrm{C}$ for $2 \mathrm{~h}$ in pooled, whole saliva $(1 \mathrm{~mL} /$ specimen $)$. They were removed and rinsed in deionised water for $5 \mathrm{~min}(1 \mathrm{~mL} /$ specimen) immediately prior to elution experiments.

Specimens were placed in UV-transparent cuvettes (Fisher Scientific, Loughborough, UK), immersed in $3 \mathrm{~mL}$ deionised water and sealed with tight-fitting lids with the 
joint wrapped with Parafilm (Bemis, Londonderry, UK). Specimens were agitated on an orbital shaker $\left(\right.$ Stuart $^{\circledR}$ SSM1; Bibby Scientific Ltd, Stone, UK) at 150 rpm. CHX concentration was measured periodically by recording absorbance at $255 \mathrm{~nm}$ using a UV spectrophotometer (U1900: Hitachi, Tokyo, Japan) and correlating with calibration standards of 5-50 $\mu \mathrm{M}$ CHX [14]. On completion of elution studies the substrates were removed from the cuvettes, blotted to remove excess water and left to dry in air. The surfaces were then imaged using SEM.

\subsection{Antimicrobial efficacy against Streptococcus gordonii}

Streptococcus gordonii colonisation of titanium substrates was investigated as a function of NP coating with and without a salivary pellicle. The proliferation of bacteria in the media in the well plates was also determined.

Yeast-nitrogen-phosphate-tryptone (YNPT) medium was prepared by mixing $1 \times$ yeast nitrogen base (Appleton Woods, Birmingham, UK), $0.05 \%$ tryptone (BD, Oxford, UK) and phosphate buffer, prepared from $10 \mathrm{mM} \mathrm{Na}_{2}$ $\mathrm{HPO}_{4}$ (Fisher Scientific, Loughborough, UK) and $\mathrm{KH}_{2} \mathrm{PO}_{4}$ (Sigma-Aldrich, Dorset, UK), pH 7.

Streptococcus gordonii DL1 cells were grown in screw cap bottles in $10 \mathrm{~mL}$ YNTP medium supplemented with $0.5 \mathrm{~mL}$ of BHY, prepared from Brain Heart Infusion (Lab M Ltd, Lancashire, UK) and Bacto Yeast Extract (BD, Oxford, UK) and $10 \mu \mathrm{L}$ of $40 \%$ aqueous glucose solution (Sigma-Aldrich) (YNPTG medium) at $37{ }^{\circ} \mathrm{C}$, overnight. The resulting culture was centrifuged and re-suspended in fresh YNTPG medium, twice. The optical density of the suspension was adjusted to $\mathrm{OD}_{600}=0.01$ (approximately $5 \times 10^{6}$ cells $/ \mathrm{mL}$ ).

Saliva from human subjects was collected and stored under approval from the National Research Ethics Committee South Central Oxford (\# 08/H0606/87+5). Pooled human saliva from at least six adult subjects, who provided written informed consent, was mixed with dithiothreitol to final concentration $2.5 \mathrm{mM}$, before being centrifuged $(12,000 \mathrm{~g}$ for $10 \mathrm{~min})$, and re-suspended at $10 \%$ solution with DI water. The saliva was then filtered through a $0.45 \mu \mathrm{m}$ filter (Sastedt, Leicester, UK) and stored at $-20{ }^{\circ} \mathrm{C}$ until required.

NP-coated and uncoated titanium substrates were placed in a 12-well microtitre plate (Greiner Bio-one, Stonehouse, UK), immersed in $1 \mathrm{~mL}$ of $10 \%$ saliva and incubated at $4{ }^{\circ} \mathrm{C}$ for $2 \mathrm{~h}$. They were then rinsed in $1 \mathrm{~mL}$ phosphate buffered saline (PBS) (Sigma-Aldrich Company Ltd, Dorset, UK) for 3 min on a see-saw rocker (Stuart ${ }^{\circledR}$ SSL4: Bibby Scientific Ltd, Stone, UK) at $20 \mathrm{rpm}$, immediately prior to microbiology assays.

Titanium specimens (NP-coated and uncoated control samples, with or without an acquired salivary pellicle) were placed into the wells of 12 -well microtitre plates. A $1 \mathrm{~mL}$ aliquot of YNTPG growth medium was added, followed by the addition of a further $1 \mathrm{~mL}$ of bacterial culture. The specimens were incubated at $37{ }^{\circ} \mathrm{C}$ while being agitated at $50 \mathrm{rpm}$. Specimens were removed at times $0,8,24,48 \mathrm{~h}$ and rinsed in fresh YNTPG medium for 3 min on a Stuart see-saw rocker at $20 \mathrm{rpm}$ before analysis. The experiment was performed three times with two specimens for each time point on each occasion, giving a total $n=6$ for each measurement time.

After each titanium specimen was removed and rinsed in fresh media, they were placed in $1 \mathrm{~mL}$ PBS and agitated using a vortex mixer for $30 \mathrm{~s}$, rested for $3 \mathrm{~min}$, and then vortex-mixed for a further $30 \mathrm{~s}$. The PBS containing released bacteria was then serially diluted by $10,10^{2}, 10^{3}$ and $10^{4}$ in fresh PBS; $20 \mu \mathrm{L}$ aliquots of the initial PBS solution and each dilution were spotted three times onto dry BHYN plates and incubated overnight. Media from the wells were also diluted and spotted onto BHYN agar plates using the same method. The resulting colony forming units (CFUs) were then counted. In order to assess statistical significance, a two-way analysis of variance (ANOVA) in combination with a post hoc Tukey's range test was performed using SPSS (IBM, Portsmouth, UK). Differences were deemed significant when $P<0.05$.

\subsection{Preparation of specimens for live/dead fluorescent imaging}

Substrates were prepared in the same way as described for the growth of the $S$. gordonii on titanium surfaces. Once rinsed in YNTPG medium, substrates were placed in the well of a new microtitre plate before being immersed in fresh YNTPG media containing $3 \mu \mathrm{L}$ of an equimolar solution of BacLight SYTO ${ }^{\circledR} 9$ green fluorescent stain and propidium iodide (Live Technologies, Paisley, UK) before a 15 min period of dark incubation. The specimens were then removed from the solution and immediately imaged using fluorescent optical microscopy (Leica DMLB: Leica Microsystems, Milton Keynes, UK). The SYTO 9 stain labelled all bacteria (green) in the population whereas the propidium iodide penetrated only those bacteria with damaged membranes (red).

\section{Results}

\subsection{Specimen characterisation}

Large nanoparticulate aggregates, smaller clusters and single nanoparticles were observed using TEM (Fig. 1). EDX data indicated the presence of $\mathrm{P}, \mathrm{Cl}$ and $\mathrm{Na}$ in areas dense in NPs, as well as $\mathrm{Cu}$ and Au which are attributed to 
the TEM grids used as a substrate. Mean NP size was $49.0 \mathrm{~nm}(\mathrm{SD} 13.1 \mathrm{~nm})$ from TEM $(\mathrm{n}=62 \mathrm{NPs})$. Porous nanoparticle deposits were observed on roughly polised titanium surfaces (Fig. 2); similar deposits were seen using atomic force microscopy (data not shown). Surface deposit was still observed after samples were immersed In deionised water for 95 days (Fig. 2).

\subsection{CHX elution from CHX-HMP NP coated titanium}

Soluble CHX was released from CHX-HMP NP-coated titanium continually throughout the experimental period (Fig. 3). Approximately $4 \times$ more CHX was released from specimens that were not coated with a salivary pellicle compared to pellicle-coated specimens. No sustained CHX release was observed from specimens which had been immersed in a $25 \mu \mathrm{M}$ CHX solution.

\subsection{Antimicrobial efficacy against Streptococcus gordonii}

The average number of bacteria present in the bacterial stock solution added to each well was $1.94 \times 10^{6} \mathrm{CFU} /$
Fig. 2 Scanning electron micrographs of uncoated titanium $(\mathbf{a}, \mathbf{b})$, titanium after CHX-HMP NP deposition (c, d), and titanium after CHX-HMP NP deposition and 95 days' immersion in deionised water $(\mathbf{e}, \mathbf{f})$

mL. S. gordonii CFUs as a function of time on titanium surfaces and in the wells containing the titanium surfaces are shown in Fig. 4. S. gordonii CFUs on CHX-HMP NPcoated titanium without a salivary pellicle decreased as a function of time, whereas CFUs on the uncoated titanium increased $10^{3}$-fold during the first $8 \mathrm{~h}$ and then remained constant. There was no statistically significant difference between the surfaces at time $0 \mathrm{~h}(P=1.000)$; the difference between the surfaces was first statistically significant at time point $8 \mathrm{~h}(P=0.01)$. CFUs in the wells containing the CHX-HMP NP-coated titanium decreased as a function of time, whereas CFUs in the uncoated substrate remained constant. Wells containing no titanium substrate exhibited very similar CFUs to those containing uncoated titanium (data not shown). There was no statistically significant difference between CFUs in the well at time $0 \mathrm{~h}$ $(P=1.000)$; the difference between the wells was first statistically significant at time point $24 \mathrm{~h}(P=0.001)$.
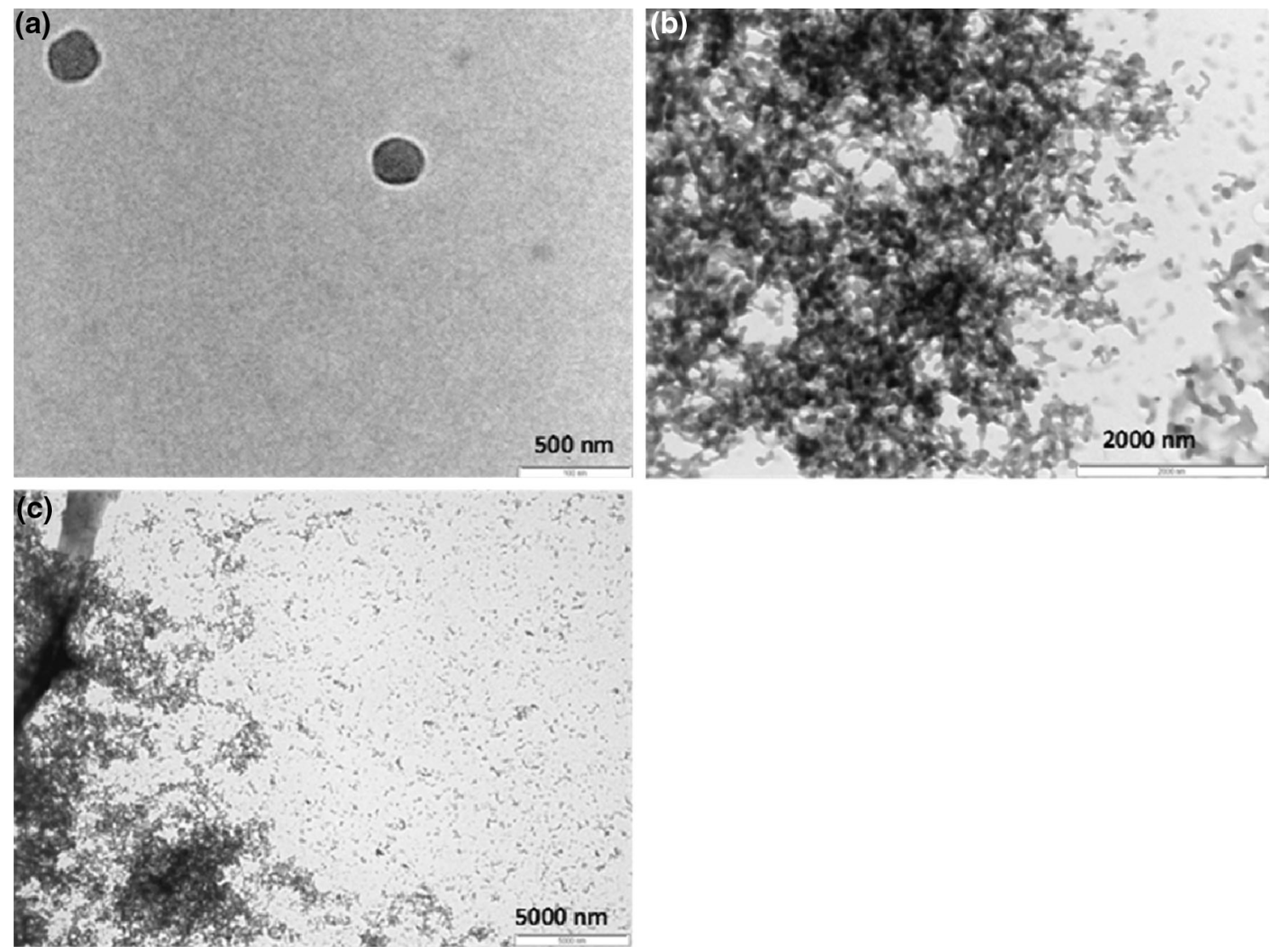

Fig. 1 Transmission electron micrographs of CHX-HMP NPs showing single nanoparticles and larger aggregates 

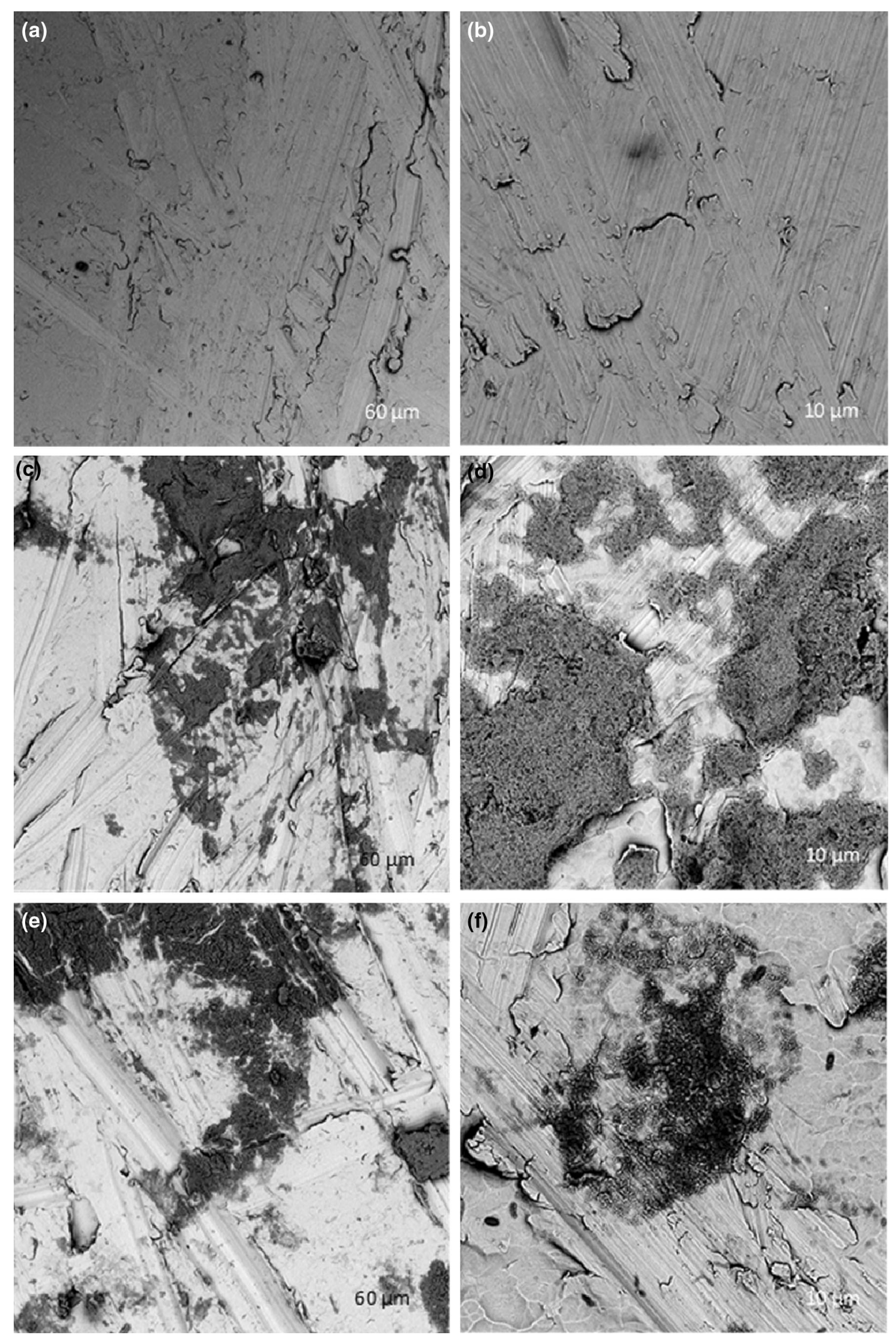
Fig. 3 Release of aqueous CHX from NP-coated titanium surfaces, with and without a salivary pellicle, compared with control samples

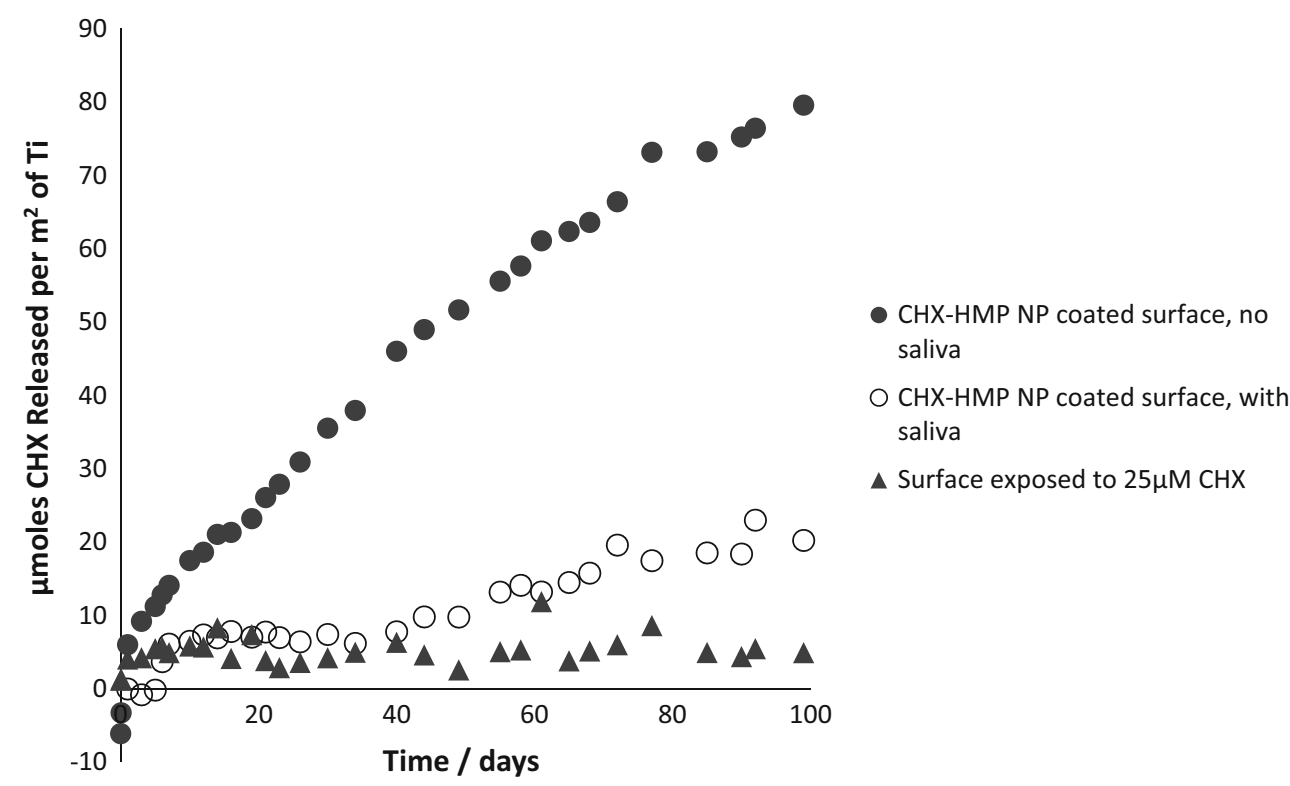

In the presence of a salivary pellicle (pellicle applied after the NP coating but prior to exposure to the bacteria), the results were broadly similar. CFUs on CHX-HMP NPcoated titanium decreased over the experimental period and were reduced to zero by $48 \mathrm{~h}$ whereas CFUs on the uncoated titanium increased 100-fold in the first $8 \mathrm{~h}$ then remained constant. There was no statistically significant difference between the surfaces at time $0 \mathrm{~h}(P=0.979)$; the difference between the surfaces was first statistically significant at time point $8 \mathrm{~h}(P=0.002)$. CFUs in the wells containing the CHX-HMP NP-coated titanium decreased with time, reaching zero by $48 \mathrm{~h}$, whereas CFUs in the uncoated substrate and blank wells remained constant. Wells containing no titanium substrate exhibited very similar CFUs to those containing uncoated titanium (data not shown). There was no statistically significant difference between CFUs in the well at time $0 \mathrm{~h}(P=1.000)$; the difference between the wells was first statistically significant at time point $24 \mathrm{~h}(P=0.012)$.

\subsection{Live/dead imaging}

There were more live bacteria on uncoated titanium substrates than on NP-coated titanium after 24 and $48 \mathrm{~h}$ (Fig. 5). This was more pronounced in the presence of an acquired pellicle, where no bacteria were observed on the NP-coated titanium or in the surrounding media after $48 \mathrm{~h}$.

\section{Discussion}

NPs with an average size of $49.0 \mathrm{~nm}$ (SD: $13.1 \mathrm{~nm}$ ) composed of CHX and HMP (indicated by the presence of $\mathrm{Cl}$ and $\mathrm{P}$ ) formed aggregated NP clusters (Fig. 1). The presence of $\mathrm{Na}$ in the EDX spectrum suggests the persistence of $\mathrm{Na}$ from the initial sodium HMP solution, most likely an artefact of drying the whole NP suspension onto the TEM grid, which contains all reaction by-products. Another reason for this could be the electrostatic attraction between the aqueous $\mathrm{Na}^{+}$ions and the negatively charged NPs, which have surface charge $-50 \mathrm{mV}$ [23].

NP aggregates adhered to titanium specimens immersed in the NP suspension forming porous micron-sized deposits surrounded by large regions of what appeared to be bare titanium (Fig. 2). This rapid attachment to the titanium surface is postulated to be due to electrostatic attraction resulting from the highly charged nature of the particles; this is further supported by the reported deposition of the same nanoparticles onto a variety of surfaces including glass and ethylene vinyl acetate (EVA), a widely used biomedical polymer [22, 23]. Nanoparticle surface deposits were observed on titanium surfaces after 95 days immersion in deionised water (Fig. 2), but there were fewer of them compared with freshly coated specimens, which is thought to be because a proportion of the coating had dissolved releasing the soluble CHX.

A sustained release of CHX was observed from CHXHMP NP-coated titanium without pellicle (Fig. 3). This compares favourably with other methods to functionalise materials with $\mathrm{CHX}$, such as a component of a polybenzyl acrylate [24] or microporous silica [25] coating where the CHX release decays rapidly over the first hours or days. CHX was also released by specimens coated with CHXHMP NPs and an overlaid salivary pellicle, at a lower rate and with larger variance in the elution data. It is thought that the passage of CHX through the pellicle is inhibited owing to the ability of the salivary pellicle to act as an ionpermeable network, inhibiting the diffusion of ions through 
Fig. 4 S. gordonii CFUs (a) on titanium surfaces and (b) in wells containing titanium surfaces, as a function of time, with error bars indicating standard deviations (a)

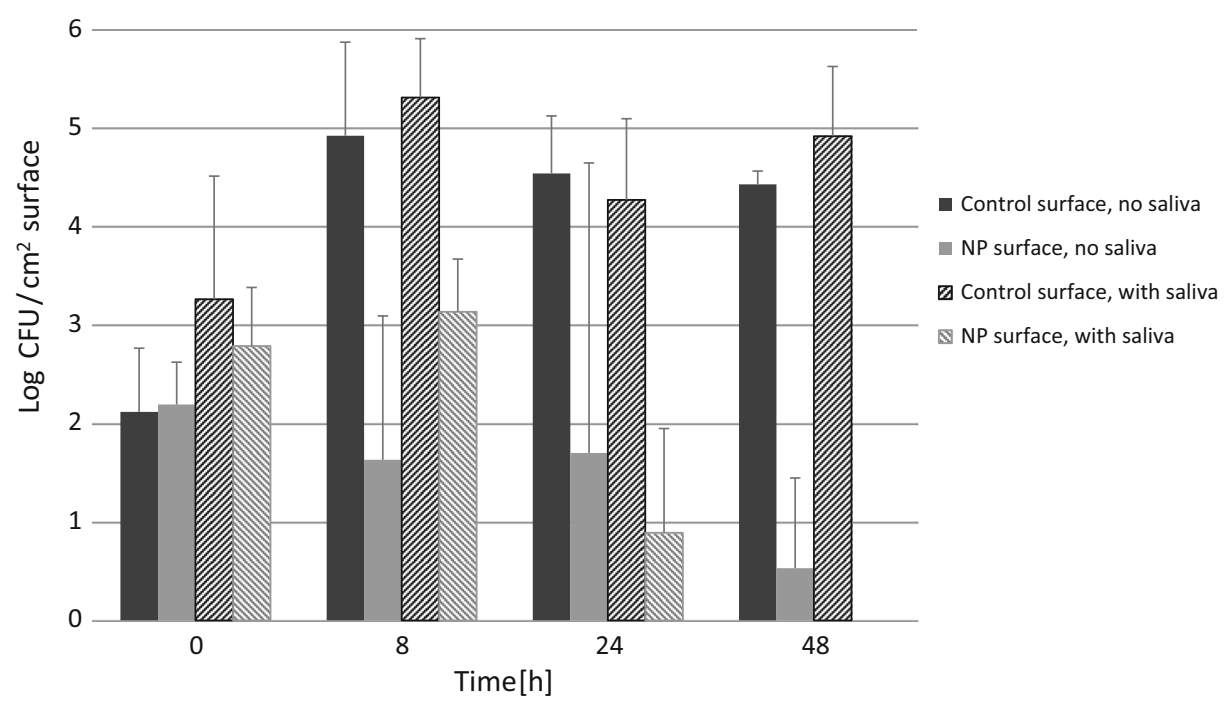

(b) 8

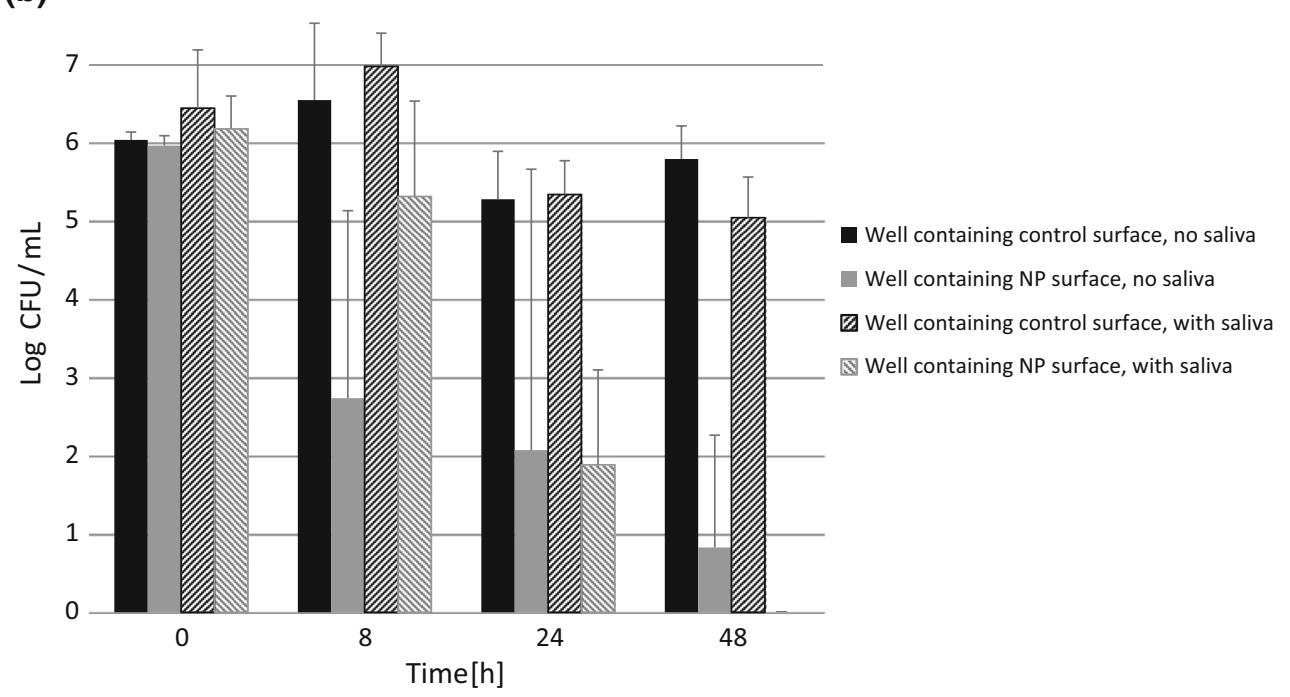

it [26]. It is possible that CHX ions are released from the NPs but remain localised at the titanium surface and/or within the pellicle film, which would explain the difference in antimicrobial activity observed in the presence of a pellicle.

At $\mathrm{t}_{0}$, there was no significant difference between CFUs of $S$. gordonii on specimens coated with CHX-HMP NP and uncoated surfaces, with and without a salivary pellicle. More bacteria adhered in the presence of a salivary pellicle, indicating the mediation of bacterial adhesion afforded by the proteinaceous film (Fig. 4). After $8 \mathrm{~h}$, CFUs on the uncoated titanium had increased 1000 -fold in the absence of a pellicle and 100-fold in its presence; the final CFU reached at $48 \mathrm{~h}$ was similar with and without the pellicle. CFUs remained stable for the NP-coated titanium but increased for uncoated titanium, both with and without a pellicle, resulting in a significant difference between the NP-coated and uncoated substrates across all time points except $t_{0}$. In the presence of a pellicle the CFUs on NPcoated surfaces decreased significantly after $24 \mathrm{~h}$, before falling again to zero for all specimens. A similar pattern was seen for the bacteria in the surrounding growth medium; these were reduced in those wells containing NPcoated specimens but not for those containing uncoated titanium. The fact that wells containing uncoated titanium and empty wells exhibited very similar numbers of bacteria indicates that the titanium per se did not affect bacterial growth in the medium. The main difference afforded by the pellicle was that viable $S$. gordonii were reduced in the absence of a pellicle but eliminated in the presence of a 

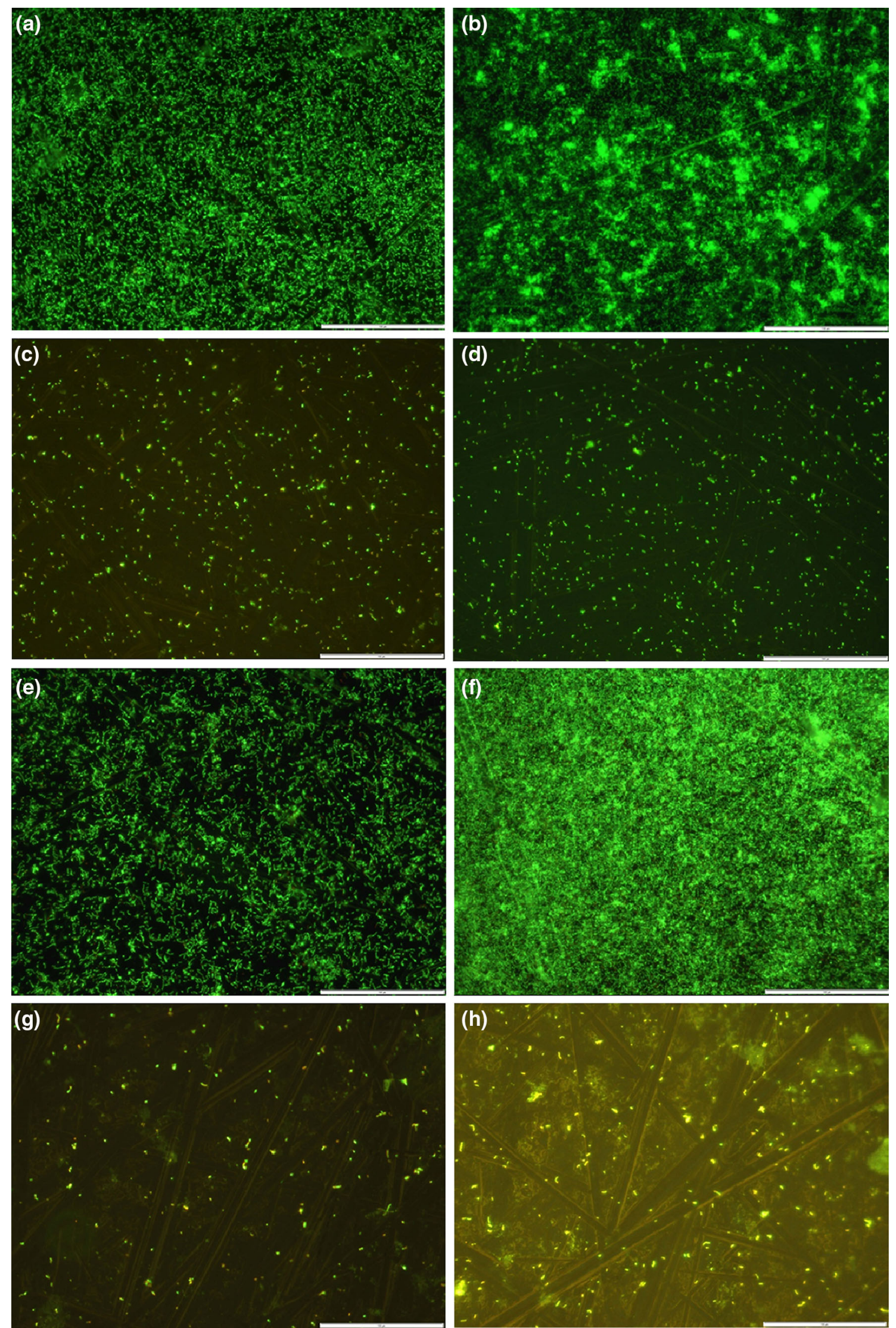
4Fig. 5 Optical micrographs showing titanium surfaces exposed to a suspension of $S$. gordonii in growth medium where $S$. gordonii is fluorescently labelled as alive (green) or dead (red). Uncoated titanium, $24 \mathrm{~h}$ (a) and $48 \mathrm{~h}$ (b) exposure; CHX-HMP NP coated titanium, $24 \mathrm{~h}$ (c) and $48 \mathrm{~h}$ (d) exposure. Uncoated titanium coated with salivary pellicle, $24 \mathrm{~h}$ (e) and $48 \mathrm{~h}$ (f) exposure; CHX-HMP NP coated titanium coated with salivary pellicle, $24 \mathrm{~h}(\mathrm{~g})$ and $48 \mathrm{~h}$ (h) exposure. Scale bar is $100 \mu \mathrm{m}$ (Color figure online)

pellicle, both on surfaces and in the medium, which suggests that active CHX was sequestered in the pellicle layer and thus offered a more concentrated source of antimicrobial for these specimens.

These observations are largely corroborated by the findings of the optical microscopy using a live/dead stain. A reduction in live bacteria was observed on the NP-coated surfaces after 24 and $48 \mathrm{~h}$, compared with uncoated surfaces (Fig. 5). The presence of some live bacteria on the NP-coated surfaces after $48 \mathrm{~h}$, when no CFUs could be retrieved, suggests that the CHX NPs were in this case offering a bacteriostatic rather than bactericidal effect. The small, but statistically significant, reduction in CFU number for the uncoated and blank wells after 24 and $48 \mathrm{~h}$ is hypothesised to be due to the reduction of nutrients in the media caused by the metabolic action of the bacteria.

Since twice-daily CHX application in situ reduced plaque formation on titanium abutments in the mouth [27], a means to apply CHX for a sustained period without regular intervention, and/or for those areas which are not accessible to a mouth rinse or other product, could have a beneficial effect with regard to biofilm formation. A randomized multi-centre clinical trial indicated that multiple applications of chlorhexidine chips over 18 weeks resulted in substantial improvement in sites with peri-implantitis, indicating that a local environment with a sustained presence of chlorhexidine was clinically beneficial in infected sites [28].

The antimicrobial efficacy demonstrated here can be expected to be sustained only as long as there remain CHX-HMP NPs to deliver soluble CHX. Since the release mechanism relies on dissolution, this will be inherently limited by the maximum NP coverage that can be applied while still allowing effective osteoblast colonisation and maturation and production of bone. The dissipation of CHX from the implant site is likely to be slower in vivo than in the experimental model reported here where the specimens are immersed in water and vigorously agitated. Irrespective, since the primary risk period for colonisation of the implant surface with microbes is during or soon after implant placement [9], and CHX offers effective treatment of peri-implant mucositis [29], a coating which could deliver CHX for weeks or months after surgery may be of utility even if the effect is not indefinite.

\section{Conclusions}

CHX-HMP NPs were used to create a porous aggregating coating on titanium surfaces. This coating released soluble CHX continually over the duration of the study. Growth of $S$. gordonii was reduced on the NP-coated surfaces compared to uncoated titanium and titanium exposed to an aqueous solution of CHX. With further optimisation, this technology may find application in the prevention and/or treatment of peri-implant infection.

Acknowledgments The authors wish to acknowledge the EPSRC for funding NJW through a Ph.D. studentship via the Bristol Centre for Functional Nanomaterials. The authors are grateful for the support of Jonathon Jones and the Electron Microscopy Unit, Lindsay Dutton, Valeria Soro and Jane Brittan in the Oral Microbiology Group, and Neil Fox, Schools of Chemistry and Physics, all at the University of Bristol.

Open Access This article is distributed under the terms of the Creative Commons Attribution 4.0 International License (http://creativecommons.org/licenses/by/4.0/), which permits unrestricted use, distribution, and reproduction in any medium, provided you give appropriate credit to the original author(s) and the source, provide a link to the Creative Commons license, and indicate if changes were made.

\section{References}

1. Le Guehennec L, Soueidan A, Layrolle P, Amouriq Y. Surface treatments of titanium dental implants for rapid osseointegration. Dent Mater. 2007;23:844-54.

2. Almaguer-Flores A, Ximenez-Fyvie LA, Rodil SE. Oral bacterial adhesion on amorphous carbon and titanium films: effect of surface roughness and culture media. J Biomed Mater Res B Appl Biomater. 2010;92:196-204. doi:10.1002/jbm.b.31506.

3. Burgers R, Gerlach T, Hahnel S, Schwarz F, Handel G, Gosau M. In vivo and in vitro biofilm formation on two different titanium implant surfaces. Clin Oral Implant Res. 2010;21:156-64. doi:10. 1111/j.1600-0501.2009.01815.x.

4. Frojd V, Chavez de Paz L, Andersson M, Wennerberg A, Davies JR, Svensater G. In situ analysis of multispecies biofilm formation on customized titanium surfaces. Mol Oral Microbiol. 2011;26:241-52. doi:10.1111/j.2041-1014.2011.00610.x.

5. Esposito M, Hirsch JM, Lekholm U, Thomsen P. Biological factors contributing to failures of osseointegrated oral implants. 1. Success criteria and epidemiology. Eur J Oral Sci. 1998;106:527-51.

6. Lindhe J, Meyle J, Group DoEWoP. Peri-implant diseases: consensus. Report of the Sixth European Workshop on Periodontology. J Clin Periodontol. 2008;35:282-5. doi:10.1111/j.1600-051X. 2008.01283.x.

7. Renvert S, Polyzois I, Persson GR. Treatment modalities for periimplant mucositis and peri-implantitis. Am J Dent. 2013;26:313-8.

8. Siqueira WL, Custodio W, McDonald EE. New insights into the composition and functions of the acquired enamel pellicle. J Dent Res. 2012;91:1110-8. doi:10.1177/0022034512462578.

9. Subramani K, Jung RE, Molenberg A, Hammerle CH. Biofilm on dental implants: a review of the literature. Int J Oral Maxillofac Implant. 2009;24:616-26.

10. Hultin M, Gustafsson A, Hallstrom H, Johansson LA, Ekfeldt A, Klinge B. Microbiological findings and host response in patients with peri-implantitis. Clin Oral Implant Res. 2002;13:349-58. 
11. Heuer W, Kettenring A, Stumpp SN, Eberhard J, Gellermann E, Winkel A, et al. Metagenomic analysis of the peri-implant and periodontal microflora in patients with clinical signs of gingivitis or mucositis. Clin Oral Investig. 2012;16:843-50. doi:10.1007/ s00784-011-0561-8.

12. Milstone AM, Passaretti CL, Perl TM. Chlorhexidine: expanding the armamentarium for infection control and prevention. Clin Infect Dis. 2008;46:274-81. doi:10.1086/524736.

13. Barbour ME, Gandhi N, el-Turki A, O’Sullivan DJ, Jagger DC. Differential adhesion of Streptococcus gordonii to anatase and rutile titanium dioxide surfaces with and without functionalization with chlorhexidine. J Biomed Mater Res A. 2009;90:993-8.

14. Barbour ME, O'Sullivan DJ, Jagger DC. Chlorhexidine adsorption to anatase and rutile titanium dioxide. Colloid Surface A. 2007;307:116-20.

15. Saidin S, Chevallier P, Abdul Kadir MR, Hermawan H, Mantovani D. Polydopamine as an intermediate layer for silver and hydroxyapatite immobilisation on metallic biomaterials surface. Mater Sci Eng C, Mater Biol Appl. 2013;33:4715-24. doi:10. 1016/j.msec.2013.07.026.

16. Wang H, Cheng M, Hu J, Wang C, Xu S, Han CC. Preparation and optimization of silver nanoparticles embedded electrospun membrane for implant associated infections prevention. ACS Appl Mater Interfaces. 2013;5:11014-21. doi:10.1021/ am403250t.

17. Xie CM, Lu X, Wang KF, Meng FZ, Jiang O, Zhang HP, et al. Silver nanoparticles and growth factors incorporated hydroxyapatite coatings on metallic implant surfaces for enhancement of osteoinductivity and antibacterial properties. ACS Appl Mater Interface. 2014; doi:10.1021/am501428e.

18. Martinez A, Guitian F, Lopez-Piriz R, Bartolome JF, Cabal B, Esteban-Tejeda L, et al. Bone loss at implant with titanium abutments coated by soda lime glass containing silver nanoparticles: a histological study in the dog. PLoS One. 2014;9:e86926. doi:10.1371/journal.pone.0086926.

19. Qureshi AT, Landry JP, Dasa V, Janes M, Hayes DJ. Can a novel silver nano coating reduce infections and maintain cell viability in vitro? J Biomater Appl. 2014;28:1028-38. doi:10.1177/ 0885328213491793.

20. De Giglio E, Cafagna D, Cometa S, Allegretta A, Pedico A, Giannossa LC, et al. An innovative, easily fabricated, silver nanoparticle-based titanium implant coating: development and analytical characterization. Anal Bioanal Chem. 2012;. doi:10. 1007/s00216-012-6293-z.
21. Pauksch L, Hartmann S, Rohnke M, Szalay G, Alt V, Schnettler $\mathrm{R}$, et al. Biocompatibility of silver nanoparticles and silver ions in primary human mesenchymal stem cells and osteoblasts. Acta Biomater. 2014;10:439-49. doi:10.1016/j.actbio.2013.09.037.

22. Barbour ME, Maddocks SE, Wood NJ, Collins AM. Synthesis, characterization, and efficacy of antimicrobial chlorhexidine hexametaphosphate nanoparticles for applications in biomedical materials and consumer products. Int $\mathrm{J}$ Nanomed. 2013;8:3507-19. doi:10.2147/IJN.S50140.

23. Wood NJ, Maddocks SE, Grady HJ, Collins AM, Barbour ME. Functionalisation of ethylene vinyl acetate with antimicrobial chlorhexidine hexametaphosphate nanoparticles. Int J Nanomed. 2014;9:4145-52.

24. Cortizo MC, Oberti TG, Cortizo MS, Cortizo AM, Fernandez Lorenzo de Mele MA. Chlorhexidine delivery system from titanium/polybenzyl acrylate coating: evaluation of cytotoxicity and early bacterial adhesion. J Dent. 2012;40:329-37. doi:10.1016/j. jdent.2012.01.008.

25. Verraedt E, Pendela M, Adams E, Hoogmartens J, Martens JA. Controlled release of chlorhexidine from amorphous microporous silica. J Control Release. 2010;142:47-52. doi:10.1016/j.jconrel. 2009.09.022.

26. Hannig C, Becker K, Hausler N, Hoth-Hannig W, Attin T, Hannig M. Protective effect of the in situ pellicle on dentin erosion—an ex vivo pilot study. Arch Oral Biol. 2007;52:444-9. doi:10.1016/j.archoralbio.2006.10.015.

27. Bressan E, Tessarolo F, Sbricoli L, Caola I, Nollo G, Di Fiore A. Effect of chlorhexidine in preventing plaque biofilm on healing abutment: a crossover controlled study. Implant Dent. 2014;23:64-8. doi:10.1097/ID.0000000000000018.

28. Machtei EE, Frankenthal S, Levi G, Elimelech R, Shoshani E, Rosenfeld O, et al. Treatment of peri-implantitis using multiple applications of chlorhexidine chips: a double-blind, randomized multi-centre clinical trial. J Clin Periodontol. 2012;39:1198-205. doi:10.1111/jcpe.12006.

29. De Siena F, Francetti L, Corbella S, Taschieri S, Del Fabbro M. Topical application of $1 \%$ chlorhexidine gel versus $0.2 \%$ mouthwash in the treatment of peri-implant mucositis. An observational study. Int J Dent Hyg. 2013;11:41-7. doi:10.1111/ idh.12002. 